\title{
Les cellules de la lignée germinale humaine ont la capacité d'internaliser la sex steroid-binding protein humaine (SBPh) : Etude par autohistoradiographie en microscopie électronique à transmission (MET)
}

\author{
R. Bedjou* ${ }^{*}$, A. Gerard**, F. Felden ${ }^{* *}$, P. FrancK***, Th. Durriez $^{\circ}$, J. Hubert ${ }^{\circ}$, \\ H. Lejeune ${ }^{\circ 0}$, J.L. Guean' $* *$, H. GerarD ${ }^{\circ}$
}

* Laboratoire d'Histologie-Embryologie-II, Morphogénèse et Reproduction, Faculté de Médecine de Nancy I, BP 184, 54505, Vandouvre-lès-Nancy, France ; ** Laboratoire de Biochimie Cellulaire et Moléculaire en Nutrition, Faculté de Médecine de Nancy I, BP 184, 54505, Vandœuvre-lès-Nancy, France ; *** Laboratoire de Biochimie Médicale I, Faculté de Médecine de Nancy I, BP 184, 54505, Vandouvre-lès-Nancy, France ; 'Service d'Endocrinologie,

CHU Brabois (Directeur Pr Leclere), 54500 Vandœuvre-Lès-Nancy, France ; ${ }^{\circ}$ Service d'Urologie CHU Brabois (Directeur Pr Mangin), 54500 Vandœuvre-Lès-Nancy, France ; ${ }^{\circ 0}$

Clinique Endocrinologique, INSERM U. 307 Hôpital Debrousse, 29, rue Sour Bouvier, 69322 Lyon Cedex 05, France.

\section{RESUME}

Il a été récemment démontré que les cellules spermatogénétiques de rat sont capables de lier et d'internaliser spécifiquement l'androgen-binding protein de rat (ABPr). De même les cellules spermatogénétiques du macaque, sont capables de lier et d'internaliser spécifiquement la sex steroid-binding protein humaine (SBPh).

Cette étude consiste à tester si des interactions entre les cellules spermatogénétiques et les protéines de liaison des stéroïdes existent également chez l'homme. Les cellules germinales proviennent d'une part des biopsies testiculaires de patients hypofertiles et d'autre part de pulpectomies indiquées pour cancer prostatique.

Les observations en MET révèlent la présence de deux types de structures reliés à l'endocytose dans les cellules spermatogénétiques. Premièrement, les puits et vésicules recouverts de 96 $\pm 10 \mathrm{~nm}$ de diamètre, associés à la membrane plasmique. Deuxièmement, les endosomes précoces (vésicules non recouvertes) de $225 \pm 60 \mathrm{~nm}$ de diamètre localisés à la périphérie du cytoplasme ainsi que les endosomes tardifs, souvent organisés en corps multivésiculaires (CMV) dans le cytoplasme périnucléaire. Ces deux types de structures de l'appareil endocytaire sont présents à tous les stades de maturation des cellules germinales. Les vésicules non recouvertes sont toujours plus nombreuses que les vésicules recouvertes quel que soit le stade.

Les cellules germinales isolées et in situ maintenues dans l'épithélium séminifère ont été exposées au milieu de culture contenant le complexe SBPh purifiée (à partir de sérum de femme enceinte) - $\Delta 6$-testotérone tritiée $(80000 \mathrm{cpm} / \mathrm{ml}, 30 \mathrm{ng})$ photomarquée. L'étude des interactions SBPh/ cellules germinales est fondée sur les 
analyses qualitatives et quantitatives du marquage révélé par autohistoradiographie en MET.

Nos résultats montrent que cette incubation provoque un important marquage des cellules spermatogénétiques. La préincubation avec de la SBPh non marquée en large excès, de même qu'un prétraitement par l'EGTA reduit significativement le marquage. Une fois internalisée, la SBPh est localisée au niveau du compartiment endocytaire et spécialement sur les membranes délimitant ce compartiment. Un marquage intranucléaire a été également observé, sauf dans les noyaux condensés des spermatides allongées.

Au total, ce travail montre que les cellules spermatogénétiques humaines possèdent les structures cellulaires caractéristiques d'une activité d'endocytose et ont la capacité de lier et d'internaliser la $\mathrm{SBPh}$ à partir du compartiment extracellulaire. Ce résultat confirme ceux obtenus sur des modèles animaux (rat et macaque) et conduit à considérer que l'existence d'interactions entre les cellules spermatogénétiques et les protéines de liaison des stéroïdes constitue un phénomène général et probablement impliqué dans le déroulement de la spermatogénèse. Les mécanismes par lesquels les proteines de liaison des stéroïdes interviennent dans le contrôle de la fertilité chez l'homme restent à élucider.

Mots Clés : $S B P h$, récepteur de la SBPh, cellules germinales, homme.

\section{INTRODUCTION}

Dans le tube séminifêre la lignée germinale est, du fait de la présence de la barrière sang-testicule, confinée dans un microenvironnemnt spécifique totalement condition- né par les cellules de Sertoli $[4,35]$. Une des fonctions majeures des cellules de Sertoli est donc d'assurer le transport de substances depuis le compartiment basal jusqu'au compartiment adluminal [36]. C'est ainsi que le fer, associé à sa protéine de liaison, la transferrine, est transporté, via le cytoplasme Sertolien, jusqu'au compartiment des cellules germinales [26, 28, 34]. L'existence d'un mécanisme similaire est suggéré pour le transfert du cuivre grâce à son transport par la céruloplasmine [26] et pour la vitamine B12 liée à la transcobalamine [2].

Concernant les protéines de liaison des stéroïdes comme l'androgen-binding protein (ABP) qui est la protéine de transport majoritaire secrétée par les cellules de Sertoli, et la première a avoir été identifiée à ce niveau $[10,19,24,39]$, leurs interactions avec la lignée germinale restaient, jusqu'à ces derniers temps, très peu étudiées. En effet, classiquement, le transport des androgènes jusqu'au contact des cellules spermatogénétiques ne semble pas être nécessaire pour leur maturation [9] et, d'ailleurs, l'existence de récepteurs des androgènes dans les cellules germinales est très controversée, si bien qu'il était généralement admis que la lignée germinale ne constituait pas une cible pour l'ABP et que le rôle principal de cette dernière était de véhiculer les androgènes, en les protégeant de la dégradation, vers des cibles androgèno-dépendantes du tractus génital, tel que l'épididyme notamment [11-13, 18, 36].

Pourtant, dés 1984, le groupe de Steinberger décrivait la présence de sites de liaison de l'ABP sur les spermatocytes chez le rat [38]. Plus récemment, nous avons montré la présence d'une activité de liaison pour l'ABP dans des extraits de membranes de cellules germinales de rat [8]. Pelliniemi a mis en évidence, chez le rat, la présence dans le cytoplasme des spermatocytes et des spermatides de granules réagissant 
positivement avec un anticorps anti-ABP [27]. Depuis, nous avons montré que les cellules germinales du macaque étaient capables, in vitro, de lier et d'internaliser la SBPh [15] et que les cellules germinales de rat était capables, in vitro, de lier et d'internaliser l'ABPr [16]. Enfin, Huang et al., ont démontré que, chez le rat hypophysectomisé, le maintien d'une activité spermatogénétique était dépendant de la présence d'ABP [21]. Au total, ces constatations convergent vers la notion que chez certaines espèces animales, les protéines de transport des stéroïdes interagissent directement avec la lignée germinale.

Le but de cette étude est de déterminer si, chez l'homme également, la lignée germinale a des propriétés de liaison membranaire et d'internalisation des protéines de transport des stéroïdes.

\section{MATERIEL \& METHODES}

\section{Matériel testiculaire humain}

Nous avons utilisé des biopsies de patients âgés de 28 à 40 ans, hypofertiles, à spermatogénèse anormale et des pulpectomies de testicules de patients porteur d'un cancer de la prostate âgés de 60 à 85 ans. Au cours de l'intervention, les prélèvements sont immédiatement placés dans du milieu de culture Ham F-12 /DMEM (1:1) contenant 2 $\mathrm{mM}$ de L-glutamine (Sigma Chemical CO., St Louis, MO) et additionné avec du pyruvate de sodium ( $1 \mathrm{mM})$ du lactate de sodium $(6 \mathrm{mM})$ et des antibiotiques (streptomycine et pénicilline), $100 \mu \mathrm{l} / \mathrm{ml}$ et 400 $\mu \mathrm{l} / \mathrm{ml}$ respectivement. Nous avons utilisé les prélèvements de 10 patients pour cette étude.

\section{Préparation des cellules germinales humaines isolées}

Dans un premier temps, les fragments de tubes séminifères sont disséqués finement afin d'éliminer l'essentiel du tissu intersti- tiel, puis collectés dans du milieu de culture frais. Les cellules germinales sont isolées soit par simple dissociation douce au Potter (Wheaton, USA) comme précédement décrit $[6,14-16]$ soit, lorsque le parenchyme présente une fibrose apparente, après un prétraitement enzymatique par une solution de collagénase/dispase (Bøhringer Mannheim, Germany) à $1 \mathrm{mg} / \mathrm{ml}$ pendant 30 à 45 $\mathrm{mn}$ à $34^{\circ} \mathrm{C}$ sous agitation. La suspension cellulaire obtenue est filtrée à travers du coton hydrophile afin de retenir les spermatozoïdes et les débris cellulaires, et centrifugée à $150 \mathrm{X} \mathrm{g}$ pendant $10 \mathrm{mn}$. Les culots cellulaires ont été récupérés et lavés deux fois avec le même milieu de culture $(150 \mathrm{X} \mathrm{g}$ pendant $10 \mathrm{mn}$ ).

La densité cellulaire de la suspension finale est déterminée au microscope à contraste de phase, et la viabilité cellulaire est estimée par le test d'exclusion au bleu trypan (Sigma). Généralement, elle est supérieure à $95 \%$. Des frottis colorés au May-Grünwald Giemsa sont réalisés pour chaque préparation afin de déterminer le degré de contamination de la préparation cellulaire par des cellules de Sertoli, de Leydig et de cellules immigrantes et de quantifier les différentes classes de maturation de cellules de la lignée germinale.

\section{Analyse cytométrique}

Les cellules germinales humaines préparées par méthode enzymo-mécanique (cf cidessus) ont été fixées dans un tampon acide citrique salé (CABS)/ethanol 100\% (1:1) [1] et analysées en cytométrie de flux pour la quantification de l'ADN. Cette dernière a été faite sur les cellules entières après marquage à l'iodure de propidium à $37^{\circ} \mathrm{C}$ pendant 1 heure et passage au cytomètre EPISC-C (Coulter, Hialeah, M.) équipé d'un laser Argon (INNOVA 90-5, CEHERENT). Analyse de la distribution des cellules correspondant aux valeurs d'ADN $1^{\prime} \mathrm{C}, 1 \mathrm{C}, 2 \mathrm{C}$ et $4 \mathrm{C}$ à l'aide d'un logiciel adapté multicycle software. 


\section{Préparation des cellules germinales in situ}

Les tubes séminifères sont séparés avec précaution du tissu interstitiel sous un stéréomicroscope, comme décrit précédemment [14-16]. Des fragments de 5 à $10 \mathrm{~mm}$ de long sont isolés. Puis, chaque fragment est ouvert selon l'axe longitudinal avec des ciseaux de microchirugie (Pacheff, Moria, France) afin d'exposer les cellules germinales adluminales au milieu de culture contenant la protéine radiomarquée.

\section{Purification de la SBPh}

$\mathrm{La} \mathrm{SBPh}$ a été purifiée à partir du sérum de femme enceinte par la méthode décrite par Egloff et al., [5]. La pureté de la SBPh est contrôlée par électrophorèse sur gel polyacrylamide, dans les conditions non dénaturantes, en présence du sulfate dodecyl de sodium (SDS-PAGE) et par immunoélectrophorèse anti-sérum de sérum humain sain. L'estimation quantitative de la $\mathrm{SBPh}$ est aussi déterminée par méthode électroimmunologique en utilisant des anticorps polyclonaux anti-SBP sérique.

\section{Couplage covalent de la $\Delta 6$-testosté- rone tritiée à la SBPh pure}

Il est réalisé selon la méthode de Taylor et al., 1980 adaptée dans notre laboratoire $[17,40]$. La $\Delta 6$-dihydrotestostérone tritiée (NEN Research Products, Boston, MA, U.S.A.) à la concentration de $20 \mathrm{nM}$ est incubée avec $0,7 \mathrm{nM}$ de $\mathrm{SBPh}$ à $4^{\circ} \mathrm{C}$ dans 1 $\mathrm{ml}$ d'une solution tris- $\mathrm{HCl} 20 \mathrm{mM}(\mathrm{pH} 8,0)$ contenant $0,15 \mathrm{M} \mathrm{NaCl}$ et $10 \%(\mathrm{v} / \mathrm{v})$ glycerol. La solution placée dans un flacon de pyrex est soumise à des irradiations successives de $15 \mathrm{mn}$, séparées par des périodes de $10 \mathrm{mn}$ dans une chambre froide $\left(4^{\circ} \mathrm{C}\right)$ pour éviter des dénaturations thermiques de la protéine. L'irradiation est réalisée à l'aide d'une lampe quartz-mercure à 450 Watts [14].

\section{Protocoles d'incubation des cellules germinales avec le complexe $\Delta 6$-testo- stérone tritiée-SBPh}

\section{a) Cellules germinales isolées}

Les cellules germinales isolées $\left(4 \times 10^{6}\right.$ cellules $/ 100 \mu$ le même milieu de culture, $\mathrm{cf}$ section 1) ont été incubées en présence du complexe SBPh-stéroïde marqué par photoaffinité ( $30 \mathrm{ng}, 80000 \mathrm{cpm} / \mathrm{ml}$ ) sous agitation douce dans un bain marie à $34^{\circ} \mathrm{C}$ pendant des délais de 30,120 et $180 \mathrm{mn}$.

Afin de déterminer la spécificité de la liaison, des cellules germinales ont été préincubées à $34^{\circ} \mathrm{C}$ en présence d'un excès $(100$ fois) de $\mathrm{SBPh}$ non marquée pendant $20 \mathrm{mn}$ avant d'ajouter le complexe SBPh-stéroïde radiomarqué pendant $30 \mathrm{mn}$ à $34^{\circ} \mathrm{C}$. L'effet des ions calciques sur la liaison du complexe $\mathrm{SBPh}$-stéroïde radiomarqué avec d'éventuels récepteurs membranaires a été étudié par une préincubation des cellules isolées dans $100 \mu \mathrm{l}$ de PBS contenant de l'EGTA (400 mM) pendant $20 \mathrm{mn}$ avant l'addition du complexe SBPh-stéroïde marqué pendant $30 \mathrm{mn}$.

A la fin des périodes d'incubation, les cellules ont été lavées avec du milieu de culture frais. Les culots cellulaires ont été recueillis par centrifugation à $150 \mathrm{Xg}$, et fixés immédiatement en vue des études en MET.

\section{b) Cellules germinales in situ}

Deux à trois fragments de tubes séminifêres ouverts ont été incubés dans $150 \mu \mathrm{l}$ de milieu de culture contenant le complexe SBPh- $\triangle 6$-testostérone marqué par photoaffinité ( $30 \mathrm{ng}, 80000 \mathrm{cpm} / \mathrm{ml}$ ) sous agitation douce dans un bain marie à $34^{\circ} \mathrm{C}$ pendant des délais de 30 et $60 \mathrm{mn}$. A la fin de la période d'incubation, les fragments sont lavés dans un milieu de culture frais et fixés pour l'étude autohistoradiographique.

Chaque condition expérimentale est faite en triple. 


\section{Préparation pour la MET}

Les échantillons ont été fixés pendant $1 \mathrm{~h}$ $30 \mathrm{mn}$ à $4^{\circ} \mathrm{C}$ dans du glutaraldéhyde (Fluka, Switzerland) 2,5\%, v/v, dans 0,1 M de tampon cacodylate de sodium, $\mathrm{pH} 7,4$ et pendant $2 \mathrm{~h}$ dans du glutaraldéhyde $5 \%$, $\mathrm{v} / \mathrm{v}$, dans le même tampon. Après lavage, les échantillons sont postfixés par le tétroxyde d'osmium $1 \%$ dans le même tampon à la température ambiante pendant 60 mn. Après déshydratation à l'éthanol, ils ont été inclus dans l'Epon 812 (Fluka, Switzerland) puis débités en coupes ultrafines $(80 \mathrm{~nm})$ avec un ultramicrotome (Ultracut E., Reichert-Jung). Avant les observations, les coupes sont contrastées par l'acétate d'uranyle et le réactif de Reynolds.

\section{Préparation pour l'autohistoradio- graphie en MET}

Des coupes de $80 \mathrm{~nm}$, recueillies sur des lames collodionnées, sont recouvertes par trempage par une émulsion nucléaire IIford L4 diluée (IIford, St Priest, France), comme décrit précédemment [11-16]. Après 2 à 3 mois d'exposition à $4^{\circ} \mathrm{C}$, les autoradiogrammes sont développés avec du MicrodolX, Kodak (Eastman Kodak Co., Rochester, NY) pour obtenir des grains filamenteux [25], et examinés en MET.

\section{Analyse du marquage}

Les grains d'argent ont été comptés directement sur les préparations observées à l'écran. Le dénombrement des grains d'argent aux grandissements X 7000 et X 12 000 a servi à calculer les densités du marquage des différentes catégories cellulaires de la lignée germinale : spermatogonies, spermatocytes et spermatides rondes et allongées. Les résultats sont exprimés en nombre de grains d'argent par $1000 \mu \mathrm{m} 2$ de section d'un type cellulaire donné. Seuls les types cellulaires clairement identifiables selon Clermont et Heller et Clermont [3, $20]$, ont été utilisés pour des comptes. La surface des coupes de cellules germinales a été calculée à partir du diamètre de la section sur les clichés de MET.

Pour chaque expérience un total d'au moins $35000 \mu \mathrm{m}^{2}$ de surface de chaque catégorie cellulaire a été exploré. Le nombre de grains d'argent par $1000 \mu \mathrm{m}^{2}$ d'Epon a été considéré comme représentatif du bruit de fond. Il a été estimé à 1,6 grains d'argent par $1000 \mu \mathrm{m}^{2}$ d'épon et systématiquement soustrait de tous les résultats.

\section{Données morphométriques}

Les diamètres de sections cellulaires, des noyaux et des organites impliqués dans l'endocytose (puits et vésicules recouverts et vésicules non recouvertes), ainsi que la distance entre le centre d'un grain d'argent et l'organite le plus proche, ont été mesurés sur les clichés pris en MET. Dans ce travail, nous avons utilisé une valeur de 145 nm comme "half distance" (HD), en accord avec la nature de l'émulsion, le type de radioélément (tritium) utilisé ainsi que l'épaisseur des coupes $(80 \mathrm{~nm})$ [31].

\section{Préparation des membranes plas- miques de cellules de la lignée germi- nale humaine}

La suspension cellulaire de la lignée germinale humaine est aliquotée en fractions de $1 \times 10^{6}$ cellules $/ m l$. Ces fractions sont broyées à vitesses croissantes dans un tampon tris- $\mathrm{HCl}(20 \mathrm{mM}) \mathrm{pH} 7,4$ dans un bain de glace pilée. Une solution contenant $1 \mu \mathrm{g}$ leupeptine $/ \mathrm{ml}, 1 \mu \mathrm{g}$ papstatine $\mathrm{A} / \mathrm{ml}$, benzamidine $0,1 \%(\mathrm{p} / \mathrm{v})$, bacitracine $0,1 \%(\mathrm{p} / \mathrm{v})$ et $0,15 \mathrm{M}$ de $\mathrm{NaCl}$ est ajoutée dans le milieu réactionnel, pour réduire la dénaturation protéique, par la libération des enzymes cellulaires. L'homogénat ainsi obtenu est centrifugé pendant $10 \mathrm{mn}$ à $1000 \mathrm{Xg}$ dans le tampon tris-HCI contenant $1 \mathrm{mM}$ de $\mathrm{CaCI} 2$ et de MgCI2. Le surnageant obtenu est centrifugé à nouveau à $27000 \mathrm{X}$ g dans le même tampon additionné de $300 \mathrm{mM}$ de mannitol. Le culot obtenu est de nouveau centrifugé dans les mêmes conditions, mais 
avec un tampon sans mannitol. Le culot obtenu est la fraction enrichie en membranes plasmiques de la lignée germinale humaine

\section{Etude de la liaison $\mathrm{SBPh} / \mathrm{mem}$ - branes plasmiques}

Le complexe SBPh-stéroïde $(0,09-1,50$ pmol) a été incubé $1 \mathrm{~h}$, à $4^{\circ} \mathrm{C}$ sous agitation douce avec les fractions enrichies en membranes plasmiques provenant de $1 \times 10^{6}$ cellules avec des concentrations croissantes de la SBPh, en présence ou en absence d'un excès de 100-fois de $\mathrm{SBPh}$ non marquée pour les expériences d'inhibition. Les parties liée et libre ont été séparées par centrifugation à $25000 \mathrm{X}$ g pendant $15 \mathrm{mn}$, et le culot a été lavé trois fois dans $20 \mathrm{mM}$ du tampon tris-HCl $(\mathrm{pH} \mathrm{7,4)}$ contenant $1 \mathrm{mM}$ $\mathrm{MgCl} 2,1 \mathrm{mM} \mathrm{CaCl} 2$ et $0,1 \%(\mathrm{p} / \mathrm{v})$ BSA. Les radioactivités du surnageant et du culot sont mesurées après addition de $3 \mathrm{ml}$ de Scintran. Chaque valeur a été déterminée à partir de trois tubes différents. La liaison spécifique a été analysée selon la méthode de Scatchard [32].

\section{Analyse statistique}

Les données expérimentales ont été exprimées dans le texte comme la moyenne \pm D.S. Les analyses statistiques concernant les différences observées entre les catégories des cellules de la lignée germinale humaine, classées selon leur stade de maturation, ont été faites par le test $t$ de Student à l'aide du logiciel StatWorks.

\section{RESULTATS}

\section{Suspensions cellulaires de la lignée germinale humaine}

\section{a) Viabilité}

Les cellules obtenues présentent généralement une viabilité supérieure à $95 \%$. Les débris cellulaires sont rares.

\section{b) Analyses sur frottis et en cytométrie de flux}

Les frottis montrent que la proportion de cellules somatiques est inférieure à $3 \%$. Les frottis, ainsi que l'analyse en cytométrie de flux montrent que tous les types cellulaires sont présents, à l'exception des spermatozoïdes matures, sur les préparations testiculaires utilisées. Cependant la proportion des différents stades de maturation varie suivant les pathologies (biopsie de patients hypofertiles). Les produits des pulpectomies présentent également un profil de répartition cellulaire anormal, généralement caractérisé par une proportion élevée des spermatogonies (Figure 1A) par rapport à un testicule d'adulte jeune (Figure 1B).

\section{Présence des structures primaires de l'appareil endocytaire}

Les structures primaires de l'appareil endocytaire sont constituées par des vésicules non recouvertes (endocytose non spécifique) et par des puits et vésicules recouverts (endocytose récepteur médiée). Ces deux types de structures sont présents à la surface de toutes les cellules de la lignée germinale humaine observées (Figures 2 à 7).

Les vésicules lisses se caractérisent par un contenu homogène et très peu dense aux électrons et par l'absence de différenciations détectables sur le versant cytosolique de l'endomembrane. Ces vésicules ont un diamètre assez régulier de $225 \pm 60 \mathrm{~nm}$. Par ailleurs, leur nombre moyen differre suivant les stades de maturation puisqu'il est respectivement de $21,6 \pm 5,0$; de $30,0 \pm 8,0$; de $20,0 \pm 3,0$ et de $33,3 \pm 9,0$ pour les spermatogonies, spermatocytes et les spermatides rondes et allongées exprimé pour $100 \mu \mathrm{m}$ de longueur de membrane (Figure 8). Ces différences ne sont pas statistiquement significatives.

Les puits et vésicules recouverts se caractérisent par un contenu plus dense aux électrons et parfois hétérogène et par la 

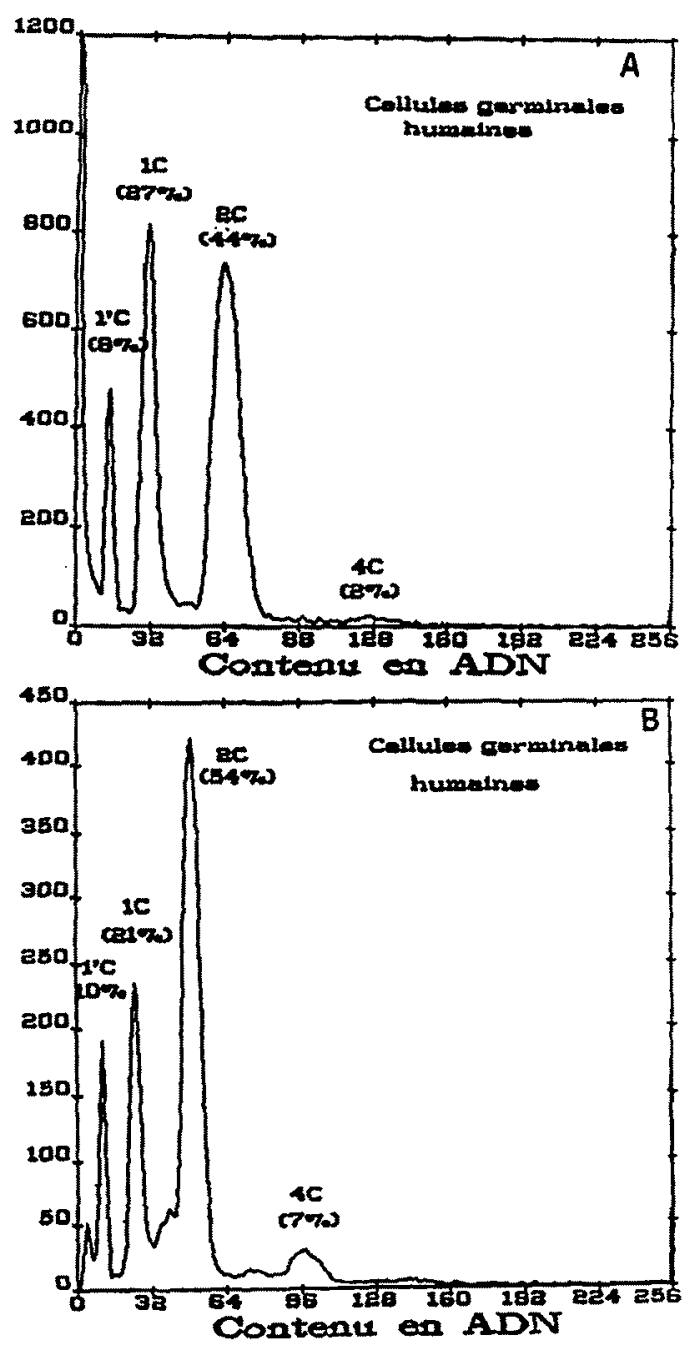

présence d'agrégats denses régulièrement disposés sur le versant cytosolique de l'endomembrane. Leur diamètre est de $96 \pm 10 \mathrm{~nm}$ pour les puits recouverts et les vésicules recouvertes. Leur nombre, exprimé par 100 $\mu \mathrm{m}$ de longueur de membrane plasmique, diffère suivant les stades de maturation puisqu'il est respectivement de $4,0 \pm 0,5 ;$ de $6,0 \pm$ 1,5 ; de $3,0 \pm 0,4$ et de $3,8 \pm 0,2$ pour les spermatogonies, les spermatocytes et les spermatides rondes et allongées (Figure 8). Cette différence est statistiquement non significative. Par contre, les différences de nombre observées entre les vésicules lisses d'une part et les structures recouvertes d'autre part, qui sont moins nombreuses, sont statistiquement significatives $(\mathrm{P} \leq 0,001)$.
Figure 1 : Etude en cytométrie de flux du contenu en ADN des suspensions de cellules germinales humaines obtenues après isolement enzymo-mécanique et filtration sur coton. A : cellules germinales d'un produit d'une pulpectomie, patient âgé de 80 ans. $B$ : pour comparaison, testicule normal, patient de 30 ans décédé accidentellement. Quatre pics distincts caractérisent les cellules germinales (1'C), (1C), (2C) et (4C). Le pic $\left(I^{\prime} C\right)$ correspond aux cellules germinales haploïdes à noyau condensé et sur spermatozoïdes. Le pic (1C) correspond aux cellules haploïdes de type : spermatides rondes. Le pic (2C) correspond aux cellules diploïdes, essentiellement : les spermatogonies interphasiques, les spermatocytes préméiotiques jusqu'au stade préleptotène exclu, les spermatocytes secondaires et les cellules somatiques interphasiques. Le pic (4C) correspond aux cellules en division qui ont déja dupliqué leur contenu d'ADN de $2 C$ à $4 C$ sont essentiellement : les spermatogonies en mitose, les spermatocytes (stade pachytène) jusqu'à l'obtention des spermatocytes secondaires.

\section{Densité de marquage}

La figure 9 montre que $30 \mathrm{mn}$ après le début de l'incubation à $34^{\circ} \mathrm{C}$ en présence du complexe $\mathrm{SBPh}$-stéroïde tritié, toutes les classes des cellules de la lignée germinale présentent un marquage très largement différent $d u$ bruit de fond puisque le nombre moyen de grains d'argent est de 36,$7 ; 19,1 ; 76,4$ et 27,9 pour $1000 \mu \mathrm{m}^{2}$ pour les spermatogonies, les spermatocytes primaires et les spermatides rondes et allongées respectivement, contre 1,6 pour $1000 \mu \mathrm{m}^{2}$ d'Epon pour le bruit de fond. Les spermatides rondes présentent la plus forte densité de marquage ( $P$ $\leq 0,001)$. Pour les délais plus longs (120 et $180 \mathrm{mn}$ ) on observe une diminution du marquage qui est statistiquement significative pour les spermatides rondes, les spermatides allongées et les spermatocytes. La figure 10 montre que la préincubation avec un excés de $\mathrm{SBPh}$ froide ou le prétraitement par l'EGTA réduisent considérablement $(\mathrm{P} \leq 0,0001)$ le marquage de ces quatres catégories cellulaires au temps $30 \mathrm{mn}$. 

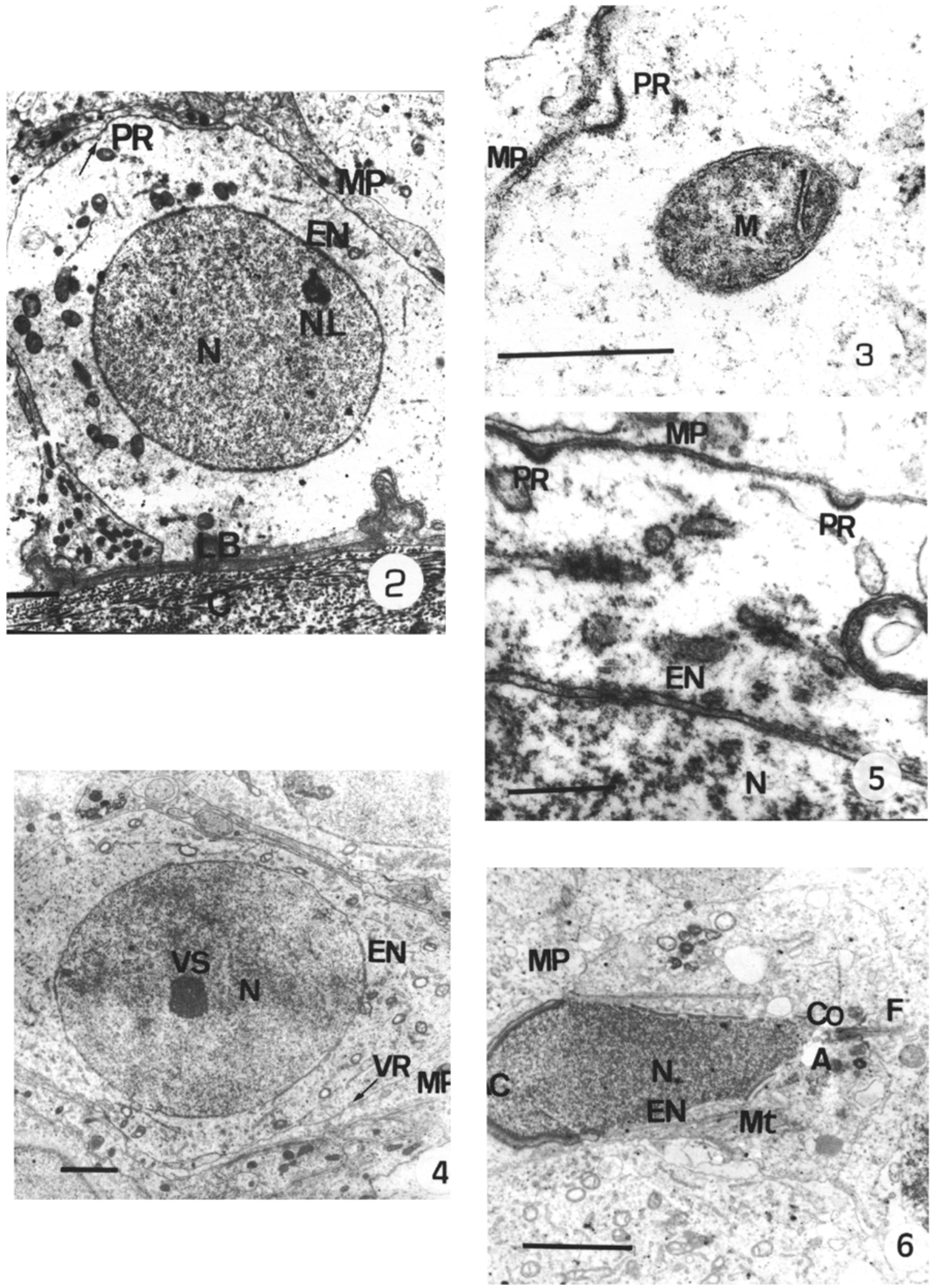


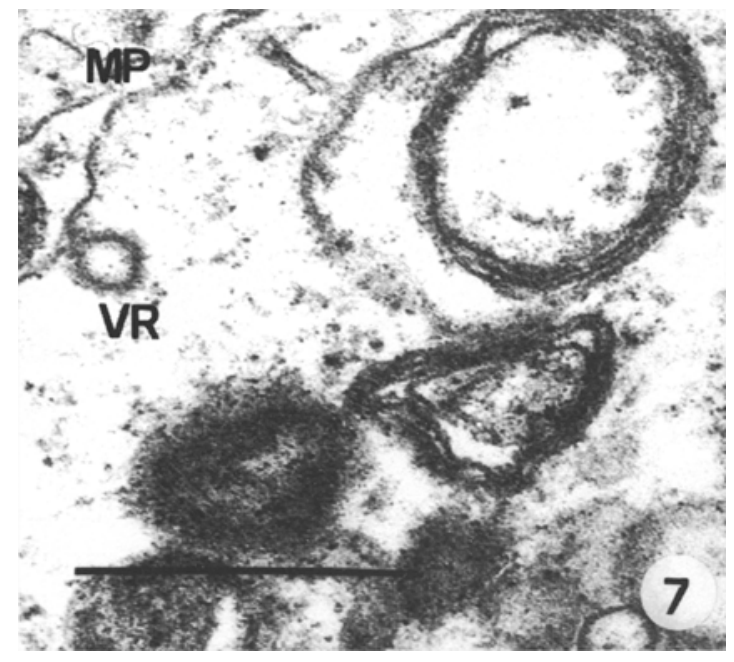

Figures 2-7: Mise en évidence des structures primaires de l'appareil endocytaire spécifique dans les cellules de la lignée germinale humaine en MET. 2-3 : Présence de puits recouverts (PR, région dense, flèche) dans une spermatogonie de type Ap in situ. $c$ : collagène; $L B$ : lame basale; $M P$ : membrane plasmique, EN : enveloppe nucléaire $N$ : noyau; $N L$ : nucléole $M$ : mitochondries $G: X 6000 ;$ Barre $1 \mu \mathrm{m}$ et $G: X 70000$; Barre $=1 \mu m .4-5:$ Présence de vésicules recouvertes (VR, flèche) et de puits recouverts (PR, région dense, flèches) dans des spermatocytes primaires pachytènes in situ. $M P$ : membrane plasmique, EN : enveloppe nucléaire $N$ : noyau, VS : vésicule sexuelle $G$ : X 6000 ; Barre $=1 \mu \mathrm{m}$ et $G: X 40000$; Barre $=1 \mu \mathrm{m} .6-7:$ Présence de vésicule recouverte (VR, région dense, flèches) dans une spermatide allongée in situ. MP : membrane plasmique, AC: acrosome, $N$ : noyau, $F$ : développement du flagelle, $\mathrm{Co}: \mathrm{col}, \mathrm{A}$ : anneau, Mt: microtubules $G: X 10000$; Barre $=1$ Hm et G: $X 80$ 000; Barre $=1 \mu \mathrm{m}$.

\section{Localisation du marquage}

Trente minutes après l'incubation à $34^{\circ} \mathrm{C}$ en présence du complexe $\Delta 6$-testotérone tritiée - $\mathrm{SBPh}$, environ $95 \%$ des grains d'argent se trouvent localisés dans le compartiment intracellulaire et 5\% sont associés à la membrane plasmique.

Au niveau de la membrane plasmique, la dimension importante des grains d'argent

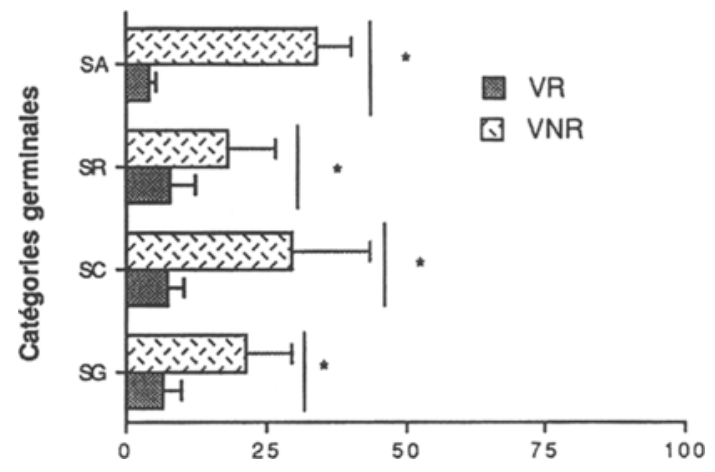

Figure 8 : Quantification des structures primaires d'endocytose (vésicules recouvertes : VR et vésicules non recouvertes : VNR) en fonction du stade de maturation des cellules spermatogénétiques germinales humaines. SG : spermatogonies, SC : spermatocytes, $S R$ : spermatides rondes, $S A$ : spermatides allongées. Les données sont exprimées en nombre moyen de VR et VNR par $100 \mu m$ de longueur de la membrane plasmique. Chaque barre représente la moyenne \pm D.S. L'étude statistique a été réalisée par le test $t$ Student. Elle a été faite entre les VR et VNR pour chaque stade de maturation $(P \leq 0,001)$.

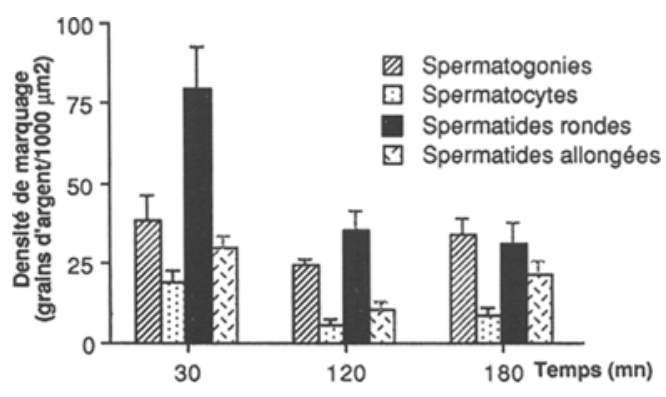

Figure 9 : Densité de marquage de chaque catégorie de cellules germinales humaines (suspension de cellules isolées). Après différents temps d'incubation en présence du complexe SBPh- $\triangle 6$-testostérone tritiée. Chaque barre représente la moyenne \pm D.S.

(250 à $350 \mathrm{~nm}$ environ) qui masquent souvent les structures sous-jacentes ne permet pas d'affirmer si la source radioactive correspond à des structures de type puits et/ou vésicules recouvertes (Figure 11). 


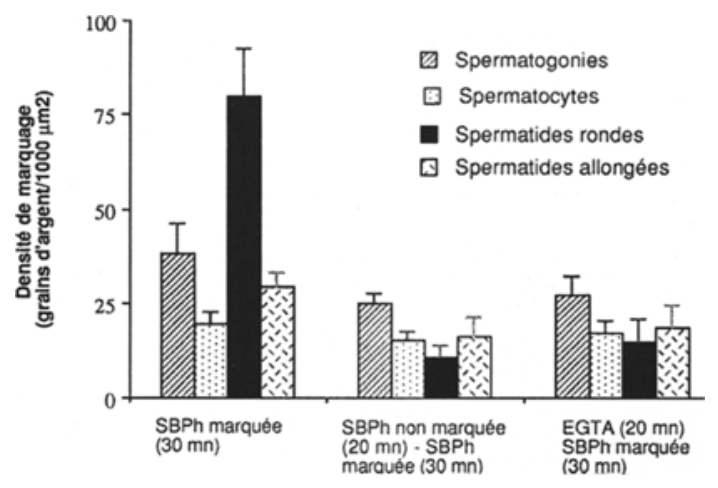

Figure 10 : Effet du prétraitement des cellules germinales par un excès de $S B P h$ non marquée et par l'EGTA sur l'intensité de l'internalisation du complexe SBPh- $\mathbf{6}$-testostérone tritiée. Les analyses quantitatives ont été réalisées en MET et exprimées en grains d'argent par unités de surface et par type cellulaire. Les données sont représentées en moyenne \pm D.S.

Au niveau du compartiment intracellulaire, nous observons des grains à la fois dans le compartiment cytoplasmique et dans le compartiment nucléaire.

Dans le compartiment cytoplasmique la majorité des grains d'argent est associée à l'appareil endocytaire et en particulier avec le compartiment des endosomes dans toutes les catégories cellulaires, et dans les CMV à partir du stade spermatide. Dans ce compartiment, les grains d'argent sont principalement associés avec la membrane qui les délimite plutôt qu'avec leur contenu.

Au niveau du noyau, le marquage principal concerne l'enveloppe nucléaire et plus rarement le noyau proprement dit (Figures 12, 13).

Au total, dans les séries observées nous n'avons pas pu mettre en évidence de différences significatives dans les répartitions intracellulaires du marquage en fonction des différents temps d'incubation. Par contre, nous avons observé des différences quant à la localisation par le marquage intranucléaire qui n'est présent que dans les stades jeunes. Nous n'avons jamais observé de marquage intranucléaire des spermatides allongées matures (à partir du stade Sc) Clermont et Heller et Clermont [3, 20].

\section{Marquage des cellules germinales in situ}

Dans le cas des cellules germinales in situ (tubes séminifères ouverts), après $30 \mathrm{mn}$ d'incubation en présence du même complexe, nous observons également un marquage important des cellules de la lignée germinale quel que soit leur stade de maturation. La localisation de ce marquage et son intensité ne semblent pas différer de celui que nous avons observé lorsque les cellules germinales sont incubées $30 \mathrm{mn}$ sous forme de suspension de cellules isolées avec le même complexe (Figure 15).

\section{Etude de la liaison SBPh/membranes plasmiques}

L'analyse de Scatchard des membranes plasmiques des cellules germinales humaines avec la SBPh a donné un Kd de $0,54 \mathrm{nM}$ et un nombre de sites/mg de protéines de 2,7.1010 (Figure 16). Cette étude montre que la SBPh est capable de se lier de façon spécifique sur un seul site de liaison membranaire.

\section{DISCUSSION}

Il a été démontré de façon occasionnelle que les cellules spermatogénétiques de différentes espèces animales étaient capables d'excercer aussi bien une endocytose non spécifique via des vésicules non recouvertes $[7,33]$ qu'une endocytose spécifique via des vésicules recouvertes $[15,16,28]$. Chez le rat des études d'immunocytochimie ont montré la présence de clathrine sur les vésicules recouvertes confirmant ainsi l'aptitude des cellules germinales à excercer une endocytose médiée par des récepteurs membranaires selon les modalités décrites pour d'autres types cellulaires [16]. 


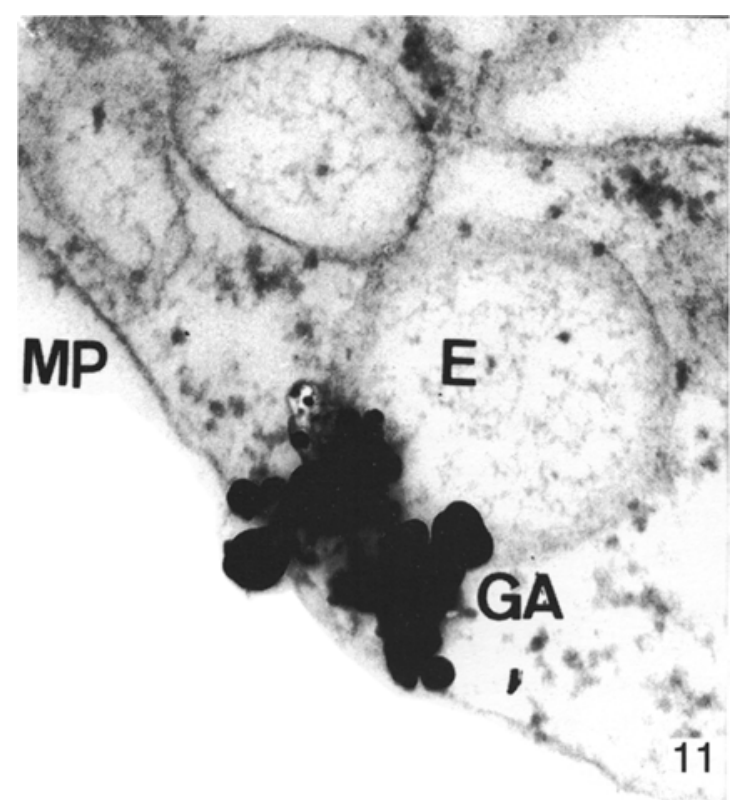

Figure 11-13 : Internalisation du complexe SBPh- 6 -testostérone tritiée dans les cellules germinales humaines (étude autoradiohistographique en microscopie électronique à transmission 2 à 3 mois d'exposition à $4^{\circ} \mathrm{C}$ et révélation au Microdol-X, Kodak). 11 : Spermatocyte primaire isolé marqué au niveau de la membrane plasmique (MP) par des grains d'argent (GA). Noter que les grains d'argent masquent le térritoire plasmique sous-jacent. $E$ : endosome. Incubation $30 \mathrm{mn}$ en présence du même complexe ligand-protéine à $34^{\circ} \mathrm{C}, G: X 80$

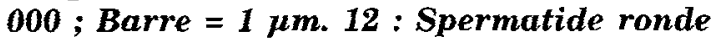
isolée (phase Golgi) marquée au niveau de la jonction entre l'enveloppe nucléaire (EN) et la vésicule acrosomiale (VA) du complexe SBPh-stéroüde tritié à $180 \mathrm{mn}$ d'incubation à $34^{\circ} \mathrm{C}, G: X 20000$; Barre= 1 Hm. 13 : Spermatocyte primaire isolé et marqué au niveau du cytoplasme profond par un grain d'argent (GA) sous forme de filament $30 \mathrm{mn}$ d'incubation au voisinage de l'enveloppe nucléaire (EN). MP : membrane plasmique, $N:$ noyau, GX:50 $000 ;$ Barre $=1 \mu \mathrm{m}$.

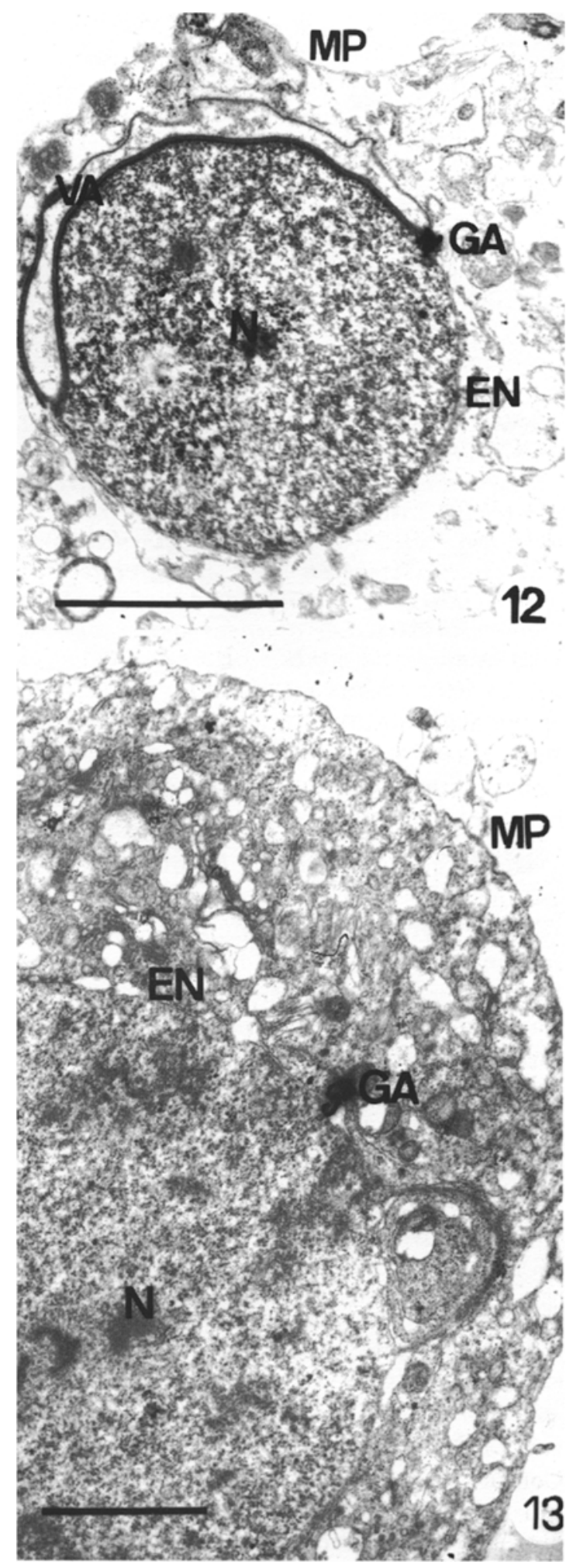




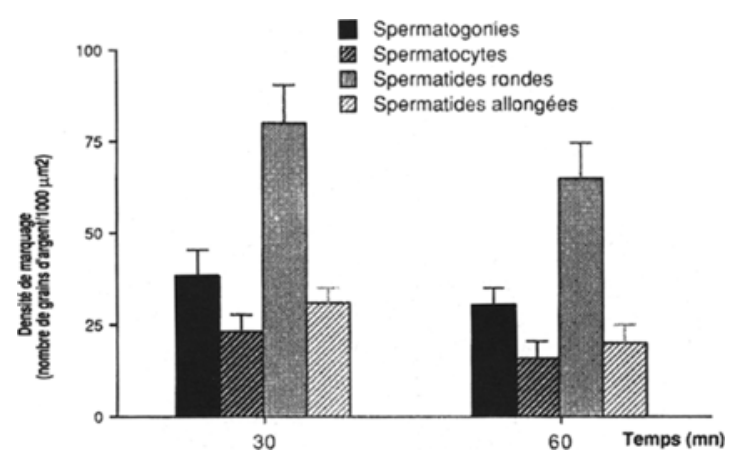

Figure 14 : Densité de marquage de chaque catégorie de cellules greminales humaines (cellules in situ dans l'épithelium séminifère). Après différents temps d'incubation en présence du complexe $\mathrm{SBPh}-\Delta 6$-testostérone tritiée. Chaque barre représente la moyenne \pm D.S.

La morphologie et la taille des vésicules recouvertes observées ici dans les cellules spermatogénétiques humaines correspond très exactement à ce type de vésicules, suggèrant par là que la lignée germinale humaine est capable de réaliser une internalisation spécifique de protéines pour lesquelles elles posséderaient le récepteur membranaire correspondant.

Nos résultats montrent effectivement que les protéines extraites des membranes de cellules germinales humaines ont une activité de liaison pour la SBPh compatible avec la présence de récepteurs pour cette protéine de transport des stéroïdes dans ces extraits. L'affinité, determinée par la représentation de Scatchard est comparable à celle observé pour le récepteur épididymaire de l'ABP [17]. La liaison SBPh-récepteur correspond morphologiquement à la détection des sources radioactives sur la membrane plasmique et dans l'appareil endocytaire des cellules spermatogénétiques lorsqu'elles ont été incubées en présence du complexe SBPh- $\Delta 6$-testotérone tritiée. Compte tenu de la taille des grains d'argent qui masquent le térritoire cellulaire sousjacent, il n'est pas possible d'affirmer que la

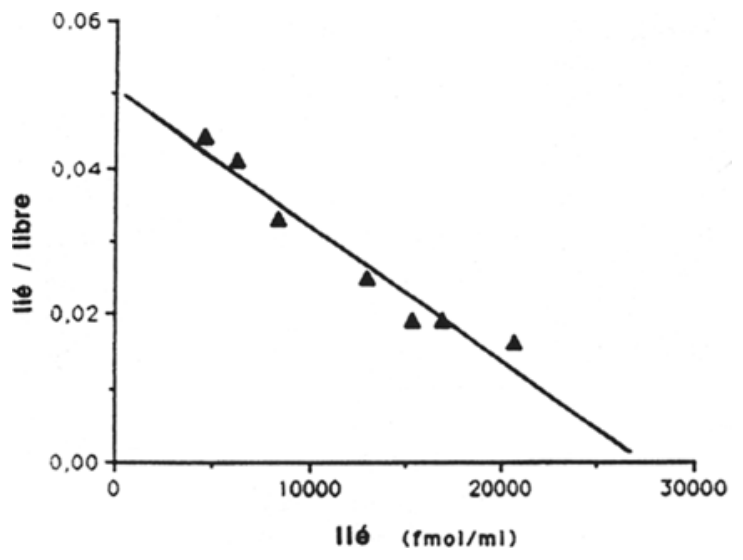

Figure 15 : Etude de récepteur membranaire à l'aide des fractions membranaires des cellules germinales en présence de la SBPh125I. Analyse par la représentation de Scatchard.

source radioactive est bien située dans des puits ou des vésicules recouvertes associée à l'internalisation récepteur médié. Cependant la réduction du marquage cellulaire par une préincubation de $30 \mathrm{mn}$ en présence de la molécule non marquée est en faveur de l'existence d'un mécanisme de liaison à un récepteur. Par ailleurs, le calcium étant nécessaire aux interactions ligand-récepteur et à la vésiculation des structures recouvertes de clathrines [22], la forte inhibition du marquage cellulaire obtenue par un prétraitement de $30 \mathrm{mn}$ par l'EGTA suggère que l'internalisation de la SBPh passe par ce type de vésicule. Enfin, même après internalisation, la $\mathrm{SBPh}$ marquée reste associée à la membrane de l'appareil endocytaire et non pas avec le contenu central des endosomes ce qui serait le cas lors d'une endocytose non spécifique de la phase fluide.

Les techniques enzymo-mécaniques utilisées pour isoler les cellules spermatogénétiques et le fait de séparer ces dernières de leurs contacts avec les cellules de Sertoli pourraient modifier temporairement le comportement du cytoplasme cortical de ces cellules. Le fait d'obtenir quantitativement 
et qualitativement le même marquage lorsque les cellules sont maintenues au sein de l'épithélium séminifère pendant l'incubation avec le complexe $\mathrm{SBPh}-\Delta 6$-testotérone tritiée montre que l'internalisation observée sur les populations de cellules isolées n'est pas liée au stress dû à la préparation des cellules.

In vivo, il n'y a pas de raison pour que la SBPh, produite majoritairement par le foie et confinée au compartiment plasmatique [30] entre en contact avec les cellules de la lignée germinale situées au delà de la barrière sang-testicule, à l'inverse de l'ABP produite par les cellules de Sertoli [10, 19, $24,39]$. Néanmoins on sait que ces protéines ont un gène commun [24] et que du point de vue de leur séquence en AA la SBPh et l'ABPr ont une homologie de $68 \%$ sur les 373 résidus qu'elles comportent l'une et l'autre [23, 24]. Il est donc probable que, dans une large mesure, les interactions entre SBP et cellules germinales soient représentatives des interactions $\mathrm{ABP}$ et cellules germinales.

Ce choix de la SBPh, qui repose sur des raisons techniques de facilité et de coût de purification par rapport à l'ABP humaine (ABPh), a déjà donné des résultats avec la lignée germinale de macaque [14]. Des expériences similaires sont en cours dans notre laboratoire avec l'ABPr et la lignée germinale humaine.

$\mathrm{Au}$ total, ces résultats chez l'homme confirment ceux obtenus chez le rat et le macaque à savoir que :

1) les cellules spermatogénétiques possèdent les structures correspondant à un appareil endocytaire permettant des internalisations spécifiques après liaison à des récepteurs membranaires,

2) les cellules spermatogénétiques internalisent par ce biais des protéines de liaison des stéroïdes. Il devient donc raisonnable d'envisager que ce phénomène ai une portée générale. Chez le rat et le macaque, il a été démontré que l'internalisation de l'ABPr et de la $\mathrm{SBPh}$, respectivement, était directement dépendante du stade de maturation de la lignée germinale [15, 16] avec notamment une accumulation nucléaire dans les stades jeunes et une accumulation cytoplasmique suggèrant que les protéines de liaison des stéroïdes pourraient contrôler certaines étapes clés de la spermatogénèse. On sait d'ailleurs qu'il y a un pic de secrétion d'ABP par les cellules de Sertoli spécifiquement chez le rat au stade VIII du cycle de l'épithélium séminifère [29].

Notre étude chez l'homme ne met en évidence aucune corrélation de ce type. Nous pensons que cette absence de corrélation résulte de la très grande disparité des prélèvements chez l'homme, contrairement à la situation de l'expérimentation animale. Il est fort probable que le fait d'utiliser des biopsies provenant de patients ayant des stérilités d'étiologies diverses ou des patients très âgés fournisse des populations de cellules germinales très disparates quant à leur état fonctionnel ce qui rend l'interprétation statistique inopérante sur des petites séries. Ceci pourrait en même temps suggérer qu’il existe des rélations entre certaines pathologies de la spermatogénèse et des troubles de la secrétion et/ou de l'internalisation de l'ABP. C'est l'hypothèse que nous cherchons à explorer actuellement dans notre laboratoire d'une part en étudiant l'activité d'internalisation de la SBPh sur des biopsies provenant de patients avec des hypofertilités d'origine connue, d'autre part en étudiant parallèlement l'expression de l'ABP dans ces biopsies, enfin en menant des études sur les anomalies de la spermatogénèse obtenues chez des souris transgéniques hyperexprimant le gène de l'ABPr (publication en préparation).

REMERCIEMENTS : Les auteurs remercient Messieurs les Professeurs A. DUPREZ, F. PLENAT, J. LECLERE et P. NABET pour leur accueil dans leurs laboratoires et Monsieur $L$. MOREL pour son assitance technique en MET. 


\section{REFERENCES}

1. ALANEN K.A., KLEMI P.J., TAILMELA S., JOENSUU H. : A simple preservative for flow cytometer DNA analysis. Cytometer, 1989, $10: 86-89$.

2. BOUKHZER E., EN NYA A., GERARD H.et al. : Transcobalamin II-Cobalamin receptors are present on rabbit germ cells. Bioch. Biophys. Acta, 1992, 1175 : 128-131.

3. CLERMONT $Y$. : The cycle of the seminiferous epithelium in man. Am. J. Anat., 1963, 118 : 509-524.

4. DYM M., FAWCETT D.M. : The bood-testis in the rat and the physiological compartmentation of the seminiferous epithelium. Biol. Reprod., 1970, 3 : 308-326.

5. EGLOFF M., VRANCKX R., TARDIVEL-LACOMBE J. et al. : Immunochemical characterization and quatification of human sex stéroid-binding plasma protein. Steroids, 1981, $37: 455-4562$.

6. EN NYA A., GUEANT J.L., NEXØ E. et al. : Endocytosis of transcobalamin in rabbit germ cells : high resolution radioautoradiographic study, 1993, $37: 353-357$.

7. FAWCETT D.M. : Interactions between Sertoli cells and germ cells. In : Male Fertility and Sterility. MANCINI R.E., MARTINI L. (Eds), Academic Press, London, 1974, 13-36.

8. FELDEN F., GUEANT J.L., EN NYA A. et al. : Photoaffinity labelled rat androgen-binding protein and human sex hormone stéroid-binding protein bind specefically to rat germ cellules. Journal of Molecular Endocrinology, 1992, $9: 39-46$.

9. FRITZ I.B. : Sites of action of androgens and follicle stimulating hormone on cells of the seminiferous tubule. In : Biochemical actions of hormones, LITWACK G. (Ed.), Academic Press, New York, 1978, 5 : 29-281.

10. FRITZ I.B., ROMMERTS F.G,, LOUIS B.G., DORRINGTON J.H. : Regulation by FSH and dbcAMP of formation of androgen-binding protein in Sertoli cellenriched cultures. J. Reprod. Fertil., 1976, 46 : 17-24.

11. GERARD A., KHANFRI J., GUEANT J.L. et al. : Electron microscope radioautographic evidence of in vivo androgen-binding protein internalization in the rat epididymis principal cells. Endocrinology, 1988a, 122 : 1297-1307.

12. GERARD H., GUEANT J.L., GERARD A. et al. : L'endocytose de l'androgen-binding protein (ABP) par les cellules principales de l'épdidyme chez le rat. Reprod. Nutr. Dev., 1988b, 28 : 1257-1266.

13. GERARD A., EL HARATE A., GUEANT J.L. et al. : Clues for receptor medieted endocytosis of intraluminal injected $A B P$ in the rat: A TEM radioautographic study. Steroids, , 1988c, $52: 239-251$.
14. GERARD A., EGLOFF M., GERARD H et al. : Internalization of human sex stéroid-binding protein in the monkey epididymis. Journal of Molecular Endocrinology, 1990, $5: 239-251$.

15. GERARD A., EN NYA A., EGLOFF M., DOMINGO M., DEGRELLE H., GERARD H. : Endocytosis of human sex steroid-binding protein in monkey germ cells. Annals of New York Academy of Sciences, 1991, $637:$ :258-276.

16. GERARD H., GERARD A., EN NYA A., FELDEN F., GUEANT J.L. : Spermatogenic cells do internalize Sertoli androgen-binding protein: A T.E.M. autoradiographic study in the rat. Endocrinology, 1994, 134 : 1515-1527.

17. GUEANT J.L., FREMONT S., KHANFRI J. et al. : Biochemical evidences for a receptor mediated uptake of rat androgen-binding protein by epididymis. Steroids, 1988, $52: 347-349$.

18. GUEANT J.L., FREMONT S., FELDEN F. et al. : Evidence that androgen-binding protein endocytosis in vitro is receptor mediated in principal cellules of the rat epididymis. Journal of Molecular Endocrinology, 1991, $7: 113-122$.

19. HAGENÄS S.L., RITZEN E.M., PLÖEN L., HANSSON V., FRENCH F.S., NAYFEH S.N. : Sertoli cell origin of testicular androgen-binding protein (ABP). Mol.Cell Endocrinol., 1975, 2 : 339-350.

20. HELLER C.G., CLERMONT Y. : Kinetics of the germinal epithelium in man. Rec. Progr. Horm. Res., 1964, $20: 545-571$.

21. HUANG H.F.S., POGACH L.M., NATHAN E. GIGLIO W., SEEBODE J.J. : Synergistic effets of follicle-stimulating hormone and testosterone on the maintenance of spermiogenesis in hypophysectomized rats : relationship with the androgen-binding protein status. Endocrinology, 1991, 128 : 3152-3161.

22. IRACE G., LIPPOLDT R.E., ELDELHOCH H., NANDI P.K. : Properties of clathrin coat struetures. Biochemistry, 1982, $9: 5764-5769$.

23. JOSEPH D.R., HALL S.H., FRENCH F.S. : Rat androgen-binding protein : Evidence for identical subunits and amino acid sequence d'homologie with human sex hormone-binding globulin. Proccedings of the National Academy of Sciences of the USA, , 1987, $84: 339-343$.

24. JOSEPH D.R., HALL S.H., CONTI M. et al. : The gene structure of the rat androgen-binding protein : identification of potential regulatory desoxyribonucleic acid elements of a follicle-stimulating hormone regulated protein. Molecular Endocrinology, 1988, $2: 3-13$.

25. KOPRIWA B.M. : A comprarison of variuos procedures for fine grain development in electron microscopic radioautography. Histochemistry, 1975, 44: 201-224. 
26. MORALES C., SYLVESTER S.R., GRISWOLD M.D. : Transport of iron and transferrin synthesis by the seminiferous epithelium of the rat in vivo. Biol. Reprod., 1987, 37 : 995-1005.

27. PELLINIEMI L.J., DYM M., GUNSALUS G.L., MUSTO N.A., BARDIN C.W., FAWCETT D.W. : Immunochemical localization of androgen-binding protein in the male reproductive tract. Endocrinology, 1981, 108 : 925-931.

28. PETRI R.G., MORALES C.R. : Receptor-mediated endocytosis of testicular transferrin by germinal cells of the rat testis. Cell Tissue Res., 1992, $267: 45-55$.

29. RITZEN E.M., BOITANI C., PARVINEN M., FRENCH F.S., FELDMAN M. : Stage dependent secretion of $A B P$ by rat seminiferous tubules. Mol. Cell Endocrinol., 1982, $25: 25-34$.

30. ROSNER W., ADEN D.P., KHAN M.S. : Hormonal influences on the secretion of stéroid-binding proteins by human hepatoma-derived cell line. J. Clin. Endocrinolo. Metab., 1984, 56 : 806-808.

31. SALPETER M.M., MCHENRY F.A., SALPETER E.E. : Resolution in electron microscope autoradiography. IV. Application to analysis of autoradiographs. J. Cell. Biol., 1978, $76: 127-130$.

32. SCATCHARD G. : The attraction of proteins for small molecules and ions. Annals of the New York . Academy of Sciences, 1949, $51: 660-672$.

33. SEGRETAIN D. : Endocytosis in spermatids during spermiogenesis of the mouse. Biol. Cell, $1989,67: 289-298$.

34. SEGRETAIN D., EGLOFF M., GERARD N., PINEAU C., JEGOU B. : Receptor-mediated and adsorptive endocytosis by male germ cells of different mammilian species. Cell Tissue Res., 1992, 268 : 471-478.

35. SETCHELL B.P., WAITES G.M.H. : The blood testis barrier. In : Greep R.O., HAMILTON D.W. (eds) Handbook of Physiology, Section 7 Endocrinology, Vol V, Male Reproduction System. Am. Physiol. Society, Washington D.C., 1975, pp 143-172.

36. SKINNER M.K. : Cell-cell interactions in the testis. Endocr. Rev., 1991, $12: 45-77$.

37. SKINNER M.K., GRISWOLD M.D. : Sertoli cells synthesize and secrete a ceruloplasmin-like protein. Biol. Reprod., 1983, 28 : 1225-1229.

38. STEINBERGER A, DIGHE R.R., DIAZ J. : Testicular peptides and their endocrine and paracrine functions. Arch. Biol. Med. Exp., 1984, 17 : 267-271.

39. STEINBERGER A, HEINDEL J.J., LINDSEY J.N., ELKINGTON J.S.H., SANBORN B.M., STEINBERGER E. : Isolation and culture of FSH responsive Sertoli cells. Endocr. Res. Commun., 1988, $2: 261-272$.
40. TAYLOR C. A., SMITH H. E., DANZO B.J. : Characterization of $A B P$ rat epididymal cytosol using a photoaffinity ligand. Journal of Biological Chemistry, 1980, $225:$ 7769-7773.

\section{ABSTRACT}

Germinal cell lineage possess the capacity of sex steroid-binding protein (hSBP) internalization : Transmission electron microscopy (TEM) autohistoradiographic study
R. Bedjou, A. Gerard, F. Felden, P.
Franck, Th. Durriez, J. Hubert, $H$.
Lejeune, J.L. GuÉant, M. Gerard

It has been recently demonstrated that rat spermatogenic cells were able to specifically bind and internalize rat androgen-binding protein (rABP) and that monkey spermatogenic cells were, in the same way, able to specifically bind and internalize human sex steroid-binding protein (hSBP).

The present study was undertaken to test if such interactions between spermatogenic cells and steroid-binding proteins do exist in the human. Germ cells were collected from testis biopsies from hypofertile patients and from testis pulpectomies from patients with prostatic cancer. TEM observations revealed the presence of two kinds of structures related to endocytosis in human spermatogenic cells. Firstly : coated pits and vesicles of $96 \pm$ $10 \mathrm{~nm}$ in diameter, associated with the plasma membrane. Secondly : early endosomes of $225 \pm 60 \mathrm{~nm}$ in diameter located in the peripheral cytoplasm and late endosomes, often organized into multivesicular bodies (MVB) in the deeper cytoplasm. Both coated and uncoated structures were equally present at all stages and uncoated structures were always more numerous than coated ones. Isolated germ cells and "in situ" germ cells maintained 
within the seminiferous epithelium were exposed to culture medium containing $80000 \mathrm{cpm} / \mathrm{ml}[3 \mathrm{H}] \Delta 6$-testosterone $(30 \mathrm{ng})$ photoaffinity-labelled hSBP purified from human late-pregnancy serum. The follow-up of labelled hSBP/germ cell interactions was based on qualitative and quantitative TEM autohistoradiography. Our observations revealed the presence of a marked labelling of spermatogenic cells. Preincubation either with excess unlabelled hSBP or pretreatment by EGTA reduced the labelling significantly. Once internalized, hSBP was found to be confined to the endocytic compartment and especially with the membrane delimitating this compartment. An intranuclear labelling was also observed which was nevertheless absent from the condensed nuclei of elongated spermatids. This leads to the hypothesis of a specific, probably receptor-mediated, endocytosis of
hSBP. This was partly confirmed by our finding that germ cell membrane extracts expressed a specific binding activity for hSBP (0.54 $\mathrm{nM}$ and $2 \times 7$ 1010 sites/mg protein).

In summary, the present study shows that human spermatogenic cells do possess active endocytic structures and have the ability to bind and internalize hSBP from the extracellular compartment. This confirm the results obtained in the rat and in the macaca and leads to propose as a general fact that steroid-binding proteins could interact with spermatogenic germ cells and to be required for the achievement of spermatogenesis. The mechanism by which "in fine" steroidbinding protein could be involved in human fertility remains to be discovered.

Key Words : $h S B P, h S B P$ receptor, germinal cell lineage, man. 